\title{
CONSEQUENCES OF SPATIAL FLUCTUATION OF COULOMB POTENTIAL IN AlGaAs WITH DX CENTERS
}

\author{
Z. WiLamowski, J. Kossut \\ Institute of Physics, Polish Academy of Sciences \\ Al. Lotników 32/46, 02-668 Warszawa, Poland \\ W. JANTSCh AND G. OSTERMAYER \\ Institut für Halbleiterphysik, Johannes-Kepler-Universität \\ 4040 Linz-Auhof, Austria
}

\begin{abstract}
Electric charges on randomly distributed impurities in semiconductors produce a spatially fluctuating potential. When the impurities are partially filled, their occupancy is not random but there appears a spatial correlation of the impurity charges appearing due to the inter-impurity Coulomb interactions. We show that when these interactions are taken into account then (i) the activation energy of the electron concentration, (ii) thermal emission kinetics, (iii) capture kinetics, (iv) persistent photoconductivity kinetics and (v) the electron mobility (in a steady state as well as during transients) in GaAlAs:Si can be explained in a consistent way. The energy diagram concerning the DX center levels with respect to minima of the conduction band as well as the capture and emission barriers (including the effect of the alloy splitting) is constructed within an approach making use of the notion of the impurity self-screening.
\end{abstract}

PACS numbers: 71.55.Dp, 71.45.Jp, 72.80.Ey

\section{Introduction}

Charged impurities in semiconductors - because of their random distribution in the crystal - give rise to a resultant potential which fluctuates in space. The dispersion of these fluctuations for perfectly random arrangement of the impurity charges is given by

$$
\sigma^{2}=\frac{2 \pi \lambda e^{4} N_{\mathrm{i}}}{\kappa^{2}},
$$


where $\lambda$ is the screening length of the potential, $\kappa$ is the dielectric constant of the material and $N_{\mathrm{i}}$ is the concentration of the charged impurities. Obviously, the fluctuation is smaller when the screening of the potential is more efficient, i.e., when the screening length $\lambda$ becomes shorter. Generally, the screening length is determined by (i) the Thomas-Fermi screening length due to free carriers and (ii) by self-screening of the impurities $[1,2]$ which is equivalent to a spatial correlation of the impurity charges. When there are only few free carriers in the material the latter mechanism of screening dominates. However, as we know from the study of $\mathrm{HgSe}: \mathrm{Fe}$ [3], the self-screening (spatial correlation) of impurity charges may be more important than the screening by free electrons even in the case of semimetals.

The correlation of the impurity charges positions can appear only when the impurity states are partially occupied by electrons. The effect of the correlations was observed as a strong reduction of the charged impurity scattering rate (high mobility values and low Dingle temperatures seen in transport studies). A simple short-range correlation model that accounted in a quantitative fashion for these anomalies in $\mathrm{HgSe}: \mathrm{Fe}$ [3] and GaAs with DX centers [4] made use of a piece-wise constant pair correlation function of charged impurities. Generally, the description of real situations requires introduction of several pair correlation functions for all types of charged centers being correlated. The number of relevant pair correlation function grows rapidly $\left(\sim n^{2}\right)$ with the number $n$ of different types of charged centers.

\section{DX centers in GaAlAs}

DX centers in GaAlAs are degenerate with the conduction band (resonant donors) provided that the Al molar fraction does not exceed $x=0.2$. They can be partially occupied by electrons and, therefore, susceptible to formation of spatially correlated state. Depending on the composition of the crystals, their level of doping and the temperature the crystals of GaAlAs can exhibit semi-insulating or semimetallic behavior. Since in these two extreme cases the screening of the impurity potentials is drastically different it is difficult to construct a common description of the correlation effects. In the first case, the screening of the potential fluctuations is almost entirely due to impurity self-screening while in the latter, free carriers play an important role in determining the screening length.

It is now established that DX state in GaAlAs represents a center with a negative on-site correlation energy $U$ [5], i.e., it binds two electrons. In this sense DX centers represent a system which is always partially occupied. In other words, DX centers are either positively (when empty) or negatively (when occupied) charged. The spatial correlation that is dominant in such circumstances is a tendency to formation of dipole-like closely spaced pairs of empty and occupied centers. Even in the simplest case with no other impurities present in the crystal, one has to consider three pair correlation functions $g_{++}(r), g_{--}(r)$ and $g_{+-}(r)$. The DX state is separated from the conduction band states by an energy barrier. Both capture and emission processes from the DX centers to the conduction band are thermally activated. Therefore, the spatial redistribution of impurity charges takes place at 
approximately $100 \mathrm{~K}$. So far the temperature effect has been neglected, however it may be of importance. One expects, of course, that the degree of correlation frozen at $100 \mathrm{~K}$ is smaller than that freezing at a lower temperature. Moreover, in the alloys the DX centers may experience a different surroundings by cations. Therefore, instead of a single value for the DX energy in GaAs, in the alloys one should deal with four different values of this quantity. We see then that, because of the alloy splitting, the number of various pair correlation functions increases to 36. At the same time, because of the differences of the energy barriers, not all DX centers may be a vailable during the formation of the correlated state of impurities. We expect then that the correlation in GaAlAs is weak, while the fluctuations of the potential can be quite large.

For these reasons the description in terms of pair correlation functions is not practical. Here we show that it is possible to describe the case of DX centers in GaAlAs via the effective screening of the impurities themselves using their (broaden by fluctuations) density of states.

\section{Formulation of the calculation scheme}

We assume that the potential due to a given impurity charge is the screened Coulomb potential with the screening length $\lambda$ given in the Thomas-Fermi approximation by

$$
\lambda^{-2}=\sum_{\alpha} \lambda_{\alpha}^{-2} \quad \text { with } \lambda_{\alpha}^{-2}=\frac{4 \pi e^{2}}{\kappa} \int \rho_{\alpha}(\varepsilon)\left(-\frac{\mathrm{d} f}{\mathrm{~d} \varepsilon}\right) \mathrm{d} \varepsilon
$$

where $\rho_{\alpha}(\varepsilon)$ is the density of states of the screening objects (impurity or band states) and $f(\varepsilon)$ is the distribution function describing the occupancy of a given state (in this way the temperature is introduced to the description). The density of impurity states can be assumed to be Gaussian centered at the bare DX center energies $E_{\mathrm{DX}}^{i}$ ( $i$ gives the number of $\mathrm{Al}$ Atoms in the DX environment) with the dispersion given by $\mathrm{Eq}$. (1). Thus the problem is a self-consistent one: in order to determine $\sigma$ one needs to know $\lambda$ and vice versa. It is not difficult to solve this problem numerically.

\section{Consequences of spatial fluctuations of the Coulomb potential in GaAlAs with DX centers}

Let us note first that the potential fluctuation $\sigma$ is typically $0.02-0.06 \mathrm{eV}$ and is of the order of the alloy splitting of the DX level. The total DOS of DX ground states is a very broad $(\sim 0.1 \mathrm{eV})$ peak without any structure. However, the fluctuation does not affect the activation energy of the emission process. As a consequence a well resolved DLTS spectra with a structure due to the alloy splitting can be observed.

- The potential fluctuation makes itself visible in several other characteristics of the system considered here. Since both the energy of DX ground state (i.e., its 
DOS) and the dispersion $\sigma$ are greater than $k T$, thus not only $E_{\mathrm{DX}}^{i}$ but also $\sigma$ determines the Fermi level and the activation energy describing the concentration of electrons in the conduction band. The second consequence of the fluctuation stems from the fact that the energy of the top of the barriers and, thus, the capture activation energy values are considerably spread out. The resulting capture transients are extremely non-exponential.

The potential fluctuation results in a low energy tail in the impurity DOS. Therefore, even when the quasi-Fermi level is much smaller than the DX level as well as smaller than the top of the barrier the electrons are effectively captured on the DX states. One can reduce this effect either by an optimization of the composition of the alloy and of the Fermi level position or by making the potential fluctuation smaller. This can be done by using pure GaAs where the fluctuation due to the alloy splitting of the DX level is absent.

Let us note finally that the correlation effects are not very strong in GaAlAs. Let us note also that in the thermal equilibrium only one alloy-split sublevel is partially filled. The remaining states are either almost completely full or completely empty ( $k T<$ alloy splitting). Thus, they do or do not take part in the screening of the potential. Under these conditions the magnitude of experimentally observed variations of the electron mobility as a function of the temperature and/or electron concentration [6] is usually smaller than several percent. There is, however, an interesting exception from this rule. When the electrons are recaptured by the DX centers after an initial illumination, it can occur that every DX sublevel is partially filled. Then, a very characteristic "two stage nature of the electron capture kinetics" [2] manifests itself as an anomalously large mobility variation. This effect is sensitively sample dependent because it results from a subtle interplay between the alloy splitting and potential fluctuations.

\section{References}

[1] T. Morgan, Phys. Rev. 134, A343 (1965).

[2] Z. Wilamowski, J. Kossut, W. Jantsch, G. Ostermayer, Proc. Winter Symp. on DX Centers, Maunterndorf, 1991, Semicond. Sci. Technol. 6, B38 (1991).

[3] Z. Wilamowski, K. Świątek, T. Dietl, J. Kossut, Solid State Commun. 74, $833(1990)$.

[4] J. Kossut, Z. Wilamowski, T. Dietl, K. Świątek, Acta Phys. Pol. A 79, 49 (1991).

[5] Z. Wilamowski, W. Jantsch, G. Ostermayer, Acta Phys. Pol. A 80, 285 (1991).

[6] R. Piotrzkowski, L. Kończewicz, E. Litwin-Staszewska, J.L. Robert, P. Lorenzini, Semicond. Sci. Technol. 6, 250 (1991). 